\title{
Elementos para un relato de la gobernanza del Sistema Vasco de Servicios Sociales
}

\section{Felix Arrieta Frutos}

Departamento de Trabajo Social y Sociología, Universidad de Deusto

$<$ felix.arrieta@deusto.es>

\section{Emma Sobremonte de Mendicuti}

Departamento de Trabajo Social y Sociología, Universidad de Deusto

Gizarte Zerbitzuen Euskal Sistema sistema gazte eta banatu bezala ikusten da askotan. Errealitate honen argitan, zeintzuk dira sistemaren gobernantza baldintzatzen duten elementuak? Nola eratu dira eta zein da sistemaren eraikuntzan izan duten eragina? Galdera hauetatik abiatuz artikulu honek elementu nagusienak bildu eta hauetatik abiatuz errelato bat osatzen du, horretarako egindako ikerketa doktoral baten analisi kualitatiboaren zati bat erabiliaz.

\section{GAKO-HITZAK:}

Gizarte-zerbitzuak, gobernantza, foralitatea, finantziazioa, herritartasuna.
El Sistema Vasco de Servicios Sociales se presenta, en multitud de ocasiones, como un sistema joven y fragmentado. A la luz de esta realidad, ¿cuáles son los elementos que determinan su gobernanza?, ¿cómo se han configurado y cuál es su influencia en la construcción del sistema? El presente artículo analiza los elementos principales para entender la gobernanza del Sistema Vasco de Servicios Sociales y configura un relato partiendo de un resumen del análisis cualitativo de una investigación doctoral realizada a tal efecto.

\section{Palabras Clave:}

Servicios sociales, gobernanza, foralidad, financiación, ciudadanía. 


\section{Introducción}

El Sistema Vasco de Servicios Sociales comienza su configuración actual, oficialmente, en diciembre de 2008, con la Ley de Servicios Sociales. Pero no ha sido un camino fácil, y su configuración, aún no finalizada, se ha encontrado con múltiples obstáculos en el camino. A priori, algunos parecían más coyunturales (crisis económica, bajada de recaudación tributaria) y otros, los más, respondían a cuestiones de más largo recorrido (sistema político institucional, reparto competencial, modelo). Todas ellas han formado parte del debate especializado desde la aprobación de la Ley.

Este artículo recoge parte del análisis y conclusiones de una investigación, desarrollada en formato de tesis doctoral, sobre la influencia de las distintas variables mencionadas a la hora de configurar los recursos residenciales para personas mayores dependientes, y sobre si esta realidad crea 0 no desigualdades territoriales (Arrieta, 2015). Las preguntas para la investigación surgían de la experiencia personal del autor, pero también de la constatación de una realidad: la dificultad de implementar las medidas previstas en la Ley $12 / 2008$.

A partir de esta constatación, la tesis doctoral analizaba indicadores cuantitativos de fuentes secundarias, que, combinados con un exhaustivo análisis cualitativo de la legislación y la normativa de cada uno de los territorios, servía de base para dilucidar si existían o no las desigualdades descritas previamente. La segunda parte del trabajo, basado en el análisis cualitativo de 35 entrevistas en profundidad realizadas a responsables políticos y técnicos de los tres niveles administrativos de la Comunidad Autónoma de Euskadi (CAE), así como a personas del tercer sector y personas expertas en la materia, ofrecía interpretaciones de los porqués de esa realidad.

Lo que exponemos en este artículo son los resultados y conclusiones del análisis cualitativo, que, ordenado de manera precisa, ofrece, tal y como hemos titulado, los elementos más importantes para entender la gobernanza del Sistema Vasco de Servicios Sociales. Aunque el trabajo de campo se realizara antes de la aprobación del Decreto de Cartera, las conclusiones que se plantean en el texto siguen siendo válidas en el contexto actual. Son variables que plantean más preguntas que respuestas, pero que nos otorgan unas cuantas pistas de a qué preguntas debería responder el Sistema a medio y largo plazo.

\section{El Sistema Vasco de Servicios Sociales}

Para entender la configuración actual del Sistema Vasco de Servicios Sociales, es necesario entender el contexto en el que se sitúa, desde el marco jurídico y la legislación española hasta las vicisitudes propias del sistema político vasco.
La Constitución Española no hace referencia, en ningún artículo concreto, al sistema de servicios sociales. Los años finales de la década de los setenta, muy prolífica en otras cuestiones, y profundamente estudiada en términos de cambio y transformación del sistema político, no fueron, sin embargo, años en los que el ámbito de los servicios sociales estuviera en la primera línea del debate público (Casado, 2007). De hecho, algunos autores apuntan que el derecho subjetivo a los servicios sociales no queda definido con claridad en el texto constitucional (Gallego, Gomá, y Subirats, 2003). El único artículo en el que se menciona el concepto 'servicios sociales' es el 50, que se refiere únicamente a los ciudadanos de la tercera edad. Aludiendo sólo al colectivo de personas mayores, ofrece una declaración incompleta de la noción de servicios sociales, tal como afirman Alemán, Alonso Seco y García Serrano (2011). Se puede deducir, por tanto, que existe una falta de reconocimiento constitucional al ámbito de los servicios sociales como sistema y conjunto de prestaciones y servicios dirigidos a toda la ciudadanía, tal y como lo conocemos en la actualidad.

En lo que concierne no ya a objeto de atención, sino a la distribución competencial, la Constitución es algo más clara. El art. 148.1.20 posibilitó la transferencia de la competencia en materia de 'asistencia social' a las comunidades autónomas, de forma explícita, como no aparece en ningún otro artículo. A este respecto, Alemán, Alonso Seco y García Serrano (2011: 43) afirman que "servicios sociales y asistencia social son conceptos diferentes, pero no está claro que el constituyente buscara esa diferenciación”. Así pues, derivado de este concepto de 'asistencia social' y de su interpretación, las comunidades autónomas comenzaron a construir su propio marco de referencia. Gallego y Subirats (2011) se unen a esta tesis al afirmar que la mayoría de las comunidades hicieron una interpretación amplia de dicho artículo, e incluso del 149.3, que permite a las comunidades autónomas asumir competencias no asignadas a ninguno de los niveles administrativos.

Así pues, se puede deducir la constitucionalizarían implícita del derecho a los servicios sociales, aunque éstos no estén recogidos como sistema jurídico autónomo (Alemán, Alonso Seco y García Serrano, 2011). Este no reconocimiento motiva, en palabras de Aznar (1994: 1.858), que “la posición jurídica de los ciudadanos no pase de (ser) un derecho condicionado y debilitado".

En segundo lugar, como normativa básica de ámbito estatal que afecta a la distribución competencial del sistema de servicios sociales, es necesario mencionar la Ley de Bases de Régimen Local, aprobada en 1985. Esta ley hace referencia a las atribuciones que tanto los municipios como las diputaciones provinciales, con la salvedad de las diputaciones forales vascas, tienen atribuidas en todo el Estado. Así, en su art. 25.2.k, atribuye a los municipios la competencia de prestar servicios sociales y de promoción y de inserción 
social. De la misma forma, en el art. 26 determina la obligatoriedad de los municipios de más de 20.000 habitantes ${ }^{1}$ de prestar dichos servicios por sí mismos 0 asociados.

Esta ley ha sido, sin embargo, modificada a través de la Ley 27/2013, de 27 de diciembre, de Racionalización y Sostenibilidad de la Administración Local. Una de las principales novedades que esta reforma introduce, es la de eliminar la competencia municipal en lo que a prestación de servicios sociales se refiere. Este elemento ha sido cuestionado por una última sentencia del Tribunal Constitucional (3 de marzo de 2016) que anula la disposición adicional que preveía la puesta en vigor de dicho precepto.

En cualquier caso, y mirando la realidad municipal desde la CAE, hay dos elementos para la reflexión. En primer lugar, la propia disposición adicional de la Ley $27 / 2013$, que hacía que el reparto competencial en materia de servicios sociales se tuviera que dilucidar en Euskadi. A este respecto, tal y como señala la circular del 11 de marzo de 2014 del Gobierno Vasco relativa a la ordenación de competencias municipales y régimen foral vasco ante la Ley 27/2013, la asignación de competencias municipales corresponde a las instituciones comunes. Y es aquí donde se plantea la segunda cuestión: la aprobación de la Ley Municipal de Euskadi, que, en su art. 17, asigna a los municipios la ordenación, planificación y gestión de los servicios sociales. Todavía habrá que esperar para ver cómo se produce este desarrollo.

\section{El sistema de servicios sociales autonómico: el Estatuto de Gernika y la Ley de Territorios Históricos}

El marco jurídico del sistema de servicios sociales autonómico se articula, en primera instancia, en relación con el Estatuto de Autonomía de Euskadi. Conocido como el Estatuto de Gernika, fue aprobado en referéndum el 25 de octubre de 1979, en un contexto social y político muy similar al de la propia Constitución Española.

El estatuto recoge, en sus arts. 10.12, 10.14 y 10.39, la competencia exclusiva en materia de asistencia social; de organización, régimen y funcionamiento de las instituciones de protección y tutela de menores, y en materia de desarrollo comunitario, condición femenina, política infantil, juvenil y de tercera edad.

Esta competencia exclusiva se definió en la Ley 27/1983, de Relaciones entre las Instituciones Comunes de la Comunidad Autónoma y los Órganos Forales de sus Territorios Históricos. Algunas autoras afirman que la conocida como Ley de Territorios Históricos (LTH) cumple las funciones de constitución interna de la $\mathrm{CAE}$, porque distribuye funciones y competencias

${ }^{1}$ En la CAE, esto es obligatorio para todos los municipios a partir de la Ley de Servicios Sociales 5/1996. del sector público entre diferentes niveles de gobierno (Gallastegui y Gallastegui, 1986; Goikoetxea, 2012). Novo (2010), sin descartar del todo esta idea, destaca también la 'modificabilidad' de la LTH por cualquier norma posterior de igual rango, puesto que el Parlamento Vasco no tiene capacidad de aprobar leyes orgánicas, que necesitan de mayorías cualificadas. Así, todas las leyes que emanan del Parlamento Vasco tienen el mismo rango normativo y, por lo tanto, cualquier ley que apruebe este mismo órgano podría modificar otra aprobada con anterioridad.

La Ley de Territorios Históricos, en su art. 7, clasifica las principales materias que pueden ser objeto de competencia de los Territorios Históricos, distinguiéndolas en tres niveles, en función de la capacidad de aquéllos de intervenir en su diseño e implementación.

- En primer lugar, el art. 7.a define las competencias exclusivas de los Territorios Históricos. Estas competencias son aquellas en las que los órganos forales ejecutan todo el proceso de las políticas públicas, desde el inicio hasta el final. Así, el control del proceso reside en la misma institución.

- En segundo lugar, el art. 7.b define las competencias en las que los Territorios Históricos tienen la capacidad de desarrollo y ejecución dentro de su territorio de la legislación emanada de los órganos comunes. En este caso, la definición de las políticas corresponde a las instituciones comunes, pero dejando también margen a las forales para introducir variables en su desarrollo, antes de su ejecución.

- En tercer lugar, el art. 7.c define aquellas competencias en las que los Territorios Históricos tendrán la potestad de ejecutar las políticas aprobadas y desarrolladas por las instituciones comunes. Ésta es la modalidad que menos margen ofrece a las instituciones forales, ya que sólo permite ejecutar lo aprobado y planificado por las instituciones comunes. Pues bien, es en este apartado en el que se encuadra la competencia de los servicios sociales:

Corresponde a los Territorios Históricos la ejecución dentro de su territorio de la legislación de las Instituciones Comunes en las siguientes materias:

1. Asistencia social, sin perjuicio de la acción directa de las Instituciones Comunes del País Vasco.

Así pues, a pesar de quedar contemplada en el apartado de menor rango competencial de todos los que la LTH prevé, la competencia de servicios sociales se ha entendido y desarrollado siempre como propia por parte de las instituciones forales. Y ésta es la pauta que se ha seguido, desde su aprobación, en toda la normativa sectorial aprobada a posteriori, y también en la acción política llevada a cabo, tal y como se verá claramente en los elementos de gobernanza que describiremos a continuación. 
Adelantado y Jiménez (2003) afirman, analizando las diversas comunidades autónomas, que el sistema de servicios sociales muestra ineficiencias en aspectos organizativos, competenciales y territoriales. Aproximándonos más a la CAE, es Fantova (2006: 11) el que afirma que hay tres elementos fundamentales a la hora de analizar el entramado institucional vasco en materia de servicios sociales:

- la "juventud" y desestructuración de la rama o sistema de servicios sociales en el entorno de la CAE;

- la dinámica de gobierno multinivel y fragmentación política en que se mueven las políticas públicas en la Comunidad Autónoma de Euskadi; y

- el vigor de la iniciativa social en el ámbito de los servicios sociales de la CAE.

Habla Fantova de juventud, porque la primera Ley vasca de Servicios Sociales (que también fue la primera de todo el Estado en ser aprobada) data de 1982. Esto es más evidente todavía, según el autor, si se compara con la legislación propia en educación y sanidad. Rodríguez Cabrero (2004) viene a reforzar esta idea con su tesis de que las políticas de bienestar españolas se desarrollan en un momento tardío, coincidiendo con un repliegue en la mayoría de países europeos, desarrollándose además con lagunas protectoras de los diversos ámbitos, entre ellos, el de los servicios sociales.

Respecto a la desestructuración, es una conclusión de Fantova (2006) a partir de la idea unánime del crecimiento desordenado como acumulación de partes. En esta misma línea, han incidido autores como Sobremonte (2009), cuando habla del sistema vasco de servicios sociales como una madeja que se ha ido tejiendo anárquicamente, y Sacanell (2009), que habla de 'caorden', es decir de un sistema concebido como concepto sociológico, pero no como realidad articulada. Duque (2012: 24) menciona esta misma idea: "no ha existido concepción de sistema único. En consecuencia, no podemos hablar de un desarrollo y despliegue homogéneo y ordenado, respondiendo a una cierta planificación". Etxezarreta (2012) también concluye que el sistema ha crecido a un ritmo vertiginoso, pero desordenado.

Esta reflexión de Sotelo (2005: 82) respecto a la aprobación del Decreto 155/2001 explica bien a las claras la situación previa a la Ley 12/2008:

si bien este Decreto 155/2001 se aprobó con el propósito de clarificar la situación y agilizar las relaciones interinstitucionales, ha fracasado en su intento. Sin duda, este fracaso se debe en parte a no permitieron ni plantear y debatir con serenidad los aspectos más controvertidos [...]. Pero en última instancia, su fracaso también es atribuible a la inadecuación de los principios funcionales de un sistema que pretende garantizar a las personas usuarias una atención integral en el marco de un continuo de servicios que se adapte a sus particulares necesidades y preferencias. Asignar la responsabilidad administrativa en función de la existencia o no de una condición de dependencia es afianzar más todavía la sectorización por colectivos y renunciar al principio de corresponsabilidad de las administraciones más cercanas al ciudadano, único capaz, en un sistema en el que interviene más de una administración, de garantizar la elección de las soluciones de atención más aptas para responder, en cada caso, a las necesidades individuales.

También Fantova achaca deficiencias de organización y coordinación a este reparto de funciones. Este diagnóstico, además de la sensación de desestructuración y agotamiento de modelo (Fantova, 2006), propició su revisión, con la puesta en marcha del proceso deliberativo que culminaría en la tercera Ley de servicios sociales de Euskadi, la Ley 12/2008.

Esta Ley surge después de que el Parlamento español aprobara, en 2006, la Ley 39/2006, de Promoción de la Autonomía Personal y Atención a las personas en situación de Dependencia. Como hemos comentado en el apartado anterior, la ley trajo consigo una nueva realidad que modificaría sustancialmente la legislación autonómica posterior: el reconocimiento del derecho subjetivo a los servicios y prestaciones reconocidos en ella. Alemán, Alonso y García (2011) afirman que ello ha producido la inclusión de estas prestaciones y servicios en las leyes autonómicas de tercera generación y que, además, estas leyes reconozcan también como derecho subjetivo prestaciones y servicios no reconocidos en la Ley 39/2006. Ésta es, en opinión de los mismos autores, la característica que más diferencia a estas leyes frente a las anteriores, y genera, además una tendencia de no retorno (ibídem: 63-64). El Real Decreto Ley 20/2012, de Medidas para Garantizar la Estabilidad Presupuestaria, ha puesto en duda tal afirmación, al establecer ya límites prácticos al desarrollo del derecho subjetivo.

La Ley 12/2008 de Servicios Sociales de Euskadi es, pues, una ley de 'tercera generación' que aporta, como principal novedad, el reconocimiento del derecho subjetivo y la universalización de los servicios. Fantova (2009) incide en estos principios y además resalta que dicha Ley busca una sinergia entre:

- La existencia de importantes responsabilidades públicas en materia de servicios sociales a nivel autonómico, foral y municipal.

- La voluntad de que el Sistema Vasco de Servicios Sociales merezca dicho nombre gracias a su unidad, articulación, coordinación y armonía. que introdujo, opuestos a los fundamentos
Para conseguir este segundo objetivo, Fantova (ibídem) enumera los mecanismos y herramientas que genera la Ley 12/2008 para la vertebración del sistema: 
- Reparto competencial y responsabilidad de una única institución.

- Estructuración de la atención en nivel primario y secundario.

- Cartera de Prestaciones y Servicios (art. 23), Plan Estratégico (art. 35) y Mapa de Servicios Sociales (art. 36).

- Órgano Interinstitucional de Servicios Sociales (art. 44).

- Criterios generales sobre la participación económica de las personas usuarias.

- Régimen de concierto.

- Observatorio Vasco de Servicios Sociales (art. 77).

- Símbolo distintivo y otras previsiones en materia de comunicación (arts. 80 y 81).

- Alta Inspección en Servicios Sociales (art. 82).

En lo que respecta al reparto competencial, la Ley enumera en sus arts. 40, 41 y 42 las funciones y competencias de cada una de las instituciones. Este nuevo reparto de funciones establece dos novedades importantes. En primer lugar, otorga al Gobierno Vasco nuevas herramientas para la coordinación general del sistema, atribución ésta que mantiene de las anteriores normativas. Además, es la primera vez que, en aplicación del principio de acción directa, el propio Gobierno participará en la provisión directa de algún servicio, como es el caso de la teleasistencia. En segundo lugar, la distinción en cuanto a la provisión entre servicios de atención primaria y atención secundaria, establecida a nivel foral y municipal, romperá con el principio de atención basado en el criterio de dependencia establecido en el Decreto 155/2001, lo que hará necesario, tal y como se menciona en la propia Ley, un periodo de ajuste. Finalmente, es necesario mencionar que la eliminación de dicho criterio y el principio de que sea una única institución la que ofrezca un único servicio también conllevará reajustes en servicios de gestión compartida hasta el momento, como el servicio de asistencia domiciliaria o los centros residenciales para personas mayores ${ }^{2}$.

En lo que respecta a la Cartera de Prestaciones y Servicios, el art. 23 de la Ley 12/2008 hace referencia a la necesidad de aprobarla como herramienta de definición de los distintos servicios y prestaciones que componen el Sistema Vasco de Servicios Sociales. Asimismo, en su art. 35 hace referencia al Plan Estratégico de Servicios Sociales, y en el 36, al Mapa. En sus disposiciones adicionales primera y segunda, la Ley establece un periodo de un año para que estos tres elementos se aprueben. Finalmente, el Decreto de Cartera fue aprobado el 6 de octubre de 2015 , con casi siete años de demora desde la aprobación de la Ley. Ésta fue una de las cuestiones,

${ }^{2}$ El debate producido en Gipuzkoa en torno a Kabia, regulado por norma foral $12 / 2014$, se sitúa en este contexto. sino la principal, que motivó la elaboración del presente artículo.

El Órgano Interinstitucional de Servicios Sociales es otra de las principales novedades de la Ley $12 / 2008$, que, en su art. 44, lo crea con el objetivo de articular la cooperación y la coordinación interinstitucional entre administraciones públicas vascas en materia de servicios sociales. Determina además que este órgano deberá contar con una composición paritaria entre la representación del Gobierno Vasco, por un lado, y la de ayuntamientos y diputaciones, por otro. El reglamento de este órgano será la primera de las cuestiones de la Ley en ser regulada 3 . Sacanell (2009) se refiere a este órgano como el "consejo de administración del sistema vasco de servicios sociales", porque es el espacio donde se produce la interacción entre las instituciones y se debate el rumbo estratégico que el sistema debe tomar.

La regulación aprobada contempla tanto el número de componentes que habrá de tener el órgano ${ }^{4}$ como la periodicidad de sus reuniones ${ }^{5}$. A este respecto, la paridad es entendida como una paridad entre el Gobierno Vasco y el resto de instituciones, siendo la suma de los representantes forales y municipales igual a los del Gobierno Vasco en dicho órgano. Además, se determina que el consejero o consejera del ámbito será quien lo presida, y que todas las decisiones se tomarán por mayoría, con excepción de la Cartera, donde habrán de contar con el visto bueno del nivel administrativo para el que se deriven obligaciones. Es decir, se introduce, sólo para la aprobación de la Cartera, el derecho de veto. Ésta es una de las cuestiones que han condicionado el funcionamiento del órgano, como veremos a lo largo de esta investigación.

El art. 57 de la Ley 12/2008 establece los principios generales respecto a la participación económica de las personas usuarias. Dicho artículo define los servicios del catálogo como gratuitos o sujetos al pago de un precio público. Establece que las personas usuarias nunca podrán participar en la financiación de los servicios y prestaciones regulados como gratuitos. Además, la Ley establece dos factores principales como base sobre la que se ponderarán los precios públicos en una regulación posterior:

- El nivel de los recursos económicos de la persona usuaria, quedando excluida la vivienda o alojamiento que constituya su residencia habitual, salvo en caso de valor excepcional. Que la Ley marque esta directriz para toda la CAE es, en sí mismo, una novedad.

${ }^{3}$ A través del Decreto 101/2010, de 30 de marzo, del Órgano Interinstitucional de Servicios Sociales.

${ }^{4}$ Seis representantes del Gobierno Vasco, de los que tres serán el consejero/consejera del ámbito de servicios sociales, el viceconsejero/a y el director o directora correspondiente; tres representantes de las diputaciones forales; y tres de los ayuntamientos, representados por la Asociación de Municipios Vascos Eudel (art. 4).

${ }^{5}$ Se establecen reuniones trimestrales (art. 7). 
- La unidad convivencial sólo se tendrá en cuenta en materia de recursos económicos en los casos en que los miembros de dicha unidad dependan económicamente de la persona beneficiaria directa del servicio.

A fecha de hoy, no existe todavía ninguna regulación específica respecto a la participación económica de las personas usuarias.

Respecto al régimen de concierto, la Ley dedica el título $\mathrm{V}$ a la participación de la iniciativa privada en la gestión de los servicios. A este respecto, obliga a que tanto la autorización y homologación de servicios como las prestaciones de primera acogida sean de gestión pública directa. Para el resto de servicios, la Ley aboga por la concertación (arts. 61-64). Además, en el art. 65 establece medidas de discriminación positiva para las entidades sin ánimo de lucro, a las que se dará prioridad en análogas condiciones. La disposición adicional octava prevé que, en el plazo de un año desde su aprobación, habrá que aprobar un decreto que regule el régimen de concierto en la CAE. Tampoco se encuentra aprobado a día de hoy.

El Observatorio Vasco de Servicios Sociales se crea en el art. 77 de la Ley 12/2008, con el objetivo de promover las medidas necesarias para la garantía y mejora de la calidad en servicios sociales. El Decreto 225/2011 regula, diseña y pone en marcha dicho observatorio, que funcionará con el nombre de Ikuspegi en el ámbito autonómico.

El art. 81 prevé la creación de un símbolo distintivo único para todo el sistema, con objeto de que la población lo identifique como tal. Mirando desde este 2016, dicho símbolo no se ha creado ni implantado en ninguno de los servicios de los que se compone el Sistema Vasco de Servicios Sociales.

Finalmente, para terminar la descripción de las características de vertebración del sistema señaladas por Fantova (2009), sólo queda mencionar la Alta Inspección de Servicios y Centros de Servicios Sociales. La Ley $12 / 2008$ crea, como una nueva medida vertebradora, la Alta Inspección y atribuye al Gobierno Vasco su competencia. Dedica, para ello, el capítulo I del título VII de la Ley (arts. 82-86). La Alta Inspección ha sido desarrollada reglamentariamente a través del Decreto 238/2010. Aun y todo, comparando las funciones que tiene asignadas y la normativa aprobada, podemos deducir que la Alta Inspección no puede estar en este momento plenamente operativa, puesto que la falta de aprobación de los criterios de concertación dificultan sido encomendadas.

El Cuadro 1 sintetiza la situación actual de los elementos descritos por Fantova como claves para la vertebración del Sistema Vasco de Servicios Sociales.

\begin{tabular}{|l|l|c|}
\hline \multicolumn{3}{|l|}{$\begin{array}{l}\text { Cuadro 1. Situación actual de los elementos vertebradores } \\
\text { del Sistema Vasco de Servicios Sociales }\end{array}$} \\
\hline Variable & Desarrollo & Situación \\
\hline Reparto competencial & Falta reajuste & $x$ \\
\hline $\begin{array}{l}\text { Atención primaria/ } \\
\text { secundaria }\end{array}$ & Falta reajuste & $x$ \\
\hline Cartera, Plan y Mapa & Decreto 185/2015 & $\checkmark$ \\
\hline Órgano interinstitucional & Decreto 101/2010 & $\checkmark$ \\
\hline $\begin{array}{l}\text { Participación económica de } \\
\text { las personas usuarias }\end{array}$ & Sin aprobar & $x$ \\
\hline Régimen de concierto & Sin aprobar & $x$ \\
\hline $\begin{array}{l}\text { Observatorio Vasco de } \\
\text { Servicios Sociales }\end{array}$ & Decreto 225/2011 & $\checkmark$ \\
\hline Símbolo distintivo & Sin desarrollar & $x$ \\
\hline Alta Inspección & Decreto 238/2010 & $\checkmark$ \\
\hline
\end{tabular}

Fuente: elaboración propia a partir de Fantova (2009).

Ésta es la realidad que motivó esta investigación, una realidad desarrollada con dificultades y de manera parcial. ¿Cuáles son las variables que han llevado a eso? ¿Se trata de la fragmentación institucional? ¿Acaso se debe a la fragmentación política? ¿Ha sido la crisis la que ha motivado esta situación? ¿Es la falta de financiación? ¿La falta de modelo? ¿El escaso recorrido de la conceptualización? Todas ellas son cuestiones que se han de tener en cuenta. Cuestiones que tejen, como veremos a continuación, el relato de la gobernanza del Sistema Vasco de Servicios Sociales.

\section{Elementos para el relato la gobernanza del Sistema}

\subsection{Federalismo-foralidad}

El federalismo o su forma de entenderlo en el sistema político vasco, la foralidad, forma parte indisoluble del relato del Sistema Vasco de Servicios Sociales. Es un elemento fundamental para entender su idiosincrasia, y todas las personas entrevistadas en el curso de este estudio se posicionaron en torno a dicho elemento. Las citas extraídas recogen algunas de estas posiciones, aunque el debate es mucho más hondo y de calado:

Yo creo en el modelo, yo sí me creo que este país está estructurado en tres niveles institucionales. Y como sí me creo eso, también me creo que, efectivamente, en la Ley de Servicios Sociales tienen que tener competencias los tres niveles; creo que, a la hora de asignación de competencias, tiene que ser en función de cómo tenemos establecida la estructura de este país. Yo creo que esto, hasta ahora, nos ha funcionado bien; por lo tanto, lo que funciona bien, soy de la opinión de no cambiar: mejorarlo, pero no cambiar (P9: 54$)^{6}$.

${ }^{6}$ En los fragmentos de entrevista empleados en este texto se utiliza la siguiente codificación: P, cargo político; T, personal técnico; S, 
¿El entramado institucional? Hombre, si hubiese sido el escenario alternativo al actual, me imagino que habría sido más poder del Gobierno [Vasco] y menos poder de las diputaciones, o [su] desaparición, ¿no? Me parece ciencia ficción ese escenario, y una reforma en esa dirección, pues también difícil, por el peso político que tienen las diputaciones (P8: 58).

Una de las personas entrevistadas se refería a la reforma de la Ley de Territorios Históricos como un escenario de ciencia ficción. Es en ese escenario donde nos situamos todavía a la hora de hablar de competencias, entramado institucional y todo aquello que pueda afectar a un concepto clave, la foralidad y, como consecuencia, el federalismo. Éste es, sin ninguna duda, el elemento clave, el más importante, para entender la gobernanza del Sistema Vasco de Servicios Sociales.

Hablar de foralidad no es hablar de cualquier cosa en Euskadi. El debate sobre cómo el elemento foral debía integrarse en la estructura institucional de la CAE rompió (formalmente) el Partido Nacionalista Vasco (PNV). Ésta es, pues, una cuestión que gravita siempre encima de cualquier persona entrevistada a la hora de dar su opinión respecto a la arquitectura institucional de un sistema de políticas públicas concreto, en este caso, el Sistema Vasco de Servicios Sociales. Tal es así que el modelo de país, lo que configura su articulación institucional, determina también el discurso sobre el Sistema Vasco de Servicios Sociales. Es más, podríamos decir que, hasta esta posición, confunde el discurso en torno al modelo de país con lo que debería ser el discurso en torno al modelo de organización del Sistema Vasco de Servicios Sociales, que, sin ser ajeno a él, podría tener elementos diferenciales.

Pero no es posible. El discurso en torno a la foralidad determina la lectura de la realidad y se convierte, sin quererlo, en un discurso de defensa del statu quo, del dejar las cosas como están, del 'virgencita, que me quede como estoy'. Bajo una aparente defensa del modelo de país, no se abordan los retos que uno u otro sistema suponen, porque 'así se decidió' o ‘yo creo en este país'. Y es aquí donde comienza un escenario con cierto aire de ciencia ficción. Porque si creemos en el país y creemos en el modelo institucional, relegando a un segundo término las necesidades a las que responden las políticas sectoriales, ¿cómo se va a articular ningún sistema alternativo al que descanse en la estructura competencial actual? La Ley de Territorios Históricos, auténtica Constitución interna de Euskadi, no lo permitiría..., simbólicamente, claro, porque, al contrario que la Constitución, tiene el mismo rango que cualquier otra ley que emane del Parlamento Vasco, como nos recuerda Novo (2010).

¿Se puede, por lo tanto, afirmar que la foralidad, y su concreción en la LTH, es un elemento clave

responsable de entidad del tercer sector; y $\mathrm{C}$, persona experta. para entender el actual Sistema Vasco de Servicios Sociales? Efectivamente, se puede. Y más allá, ¿se puede afirmar que la LTH determina y condiciona el desarrollo del Sistema Vasco de Servicios Sociales? Efectivamente, también se puede. El reparto competencial recogido en las últimas leyes de servicios sociales vascas (1996 y 2008) no responde a una realidad pensada a partir de la lectura de las necesidades de las personas que deben ser objeto de atención. Tampoco, incluso, a lo que el propio sistema debería demandar al legislador. Responde, en última instancia, a lo recogido en 1983.

Nos encontramos, por lo tanto, ante una odisea vasca en el 'espacio' de la Ley de Territorios Históricos. En un espacio de juego que se ha mantenido constante durante los últimos treinta años. Ello hace que este reparto no parezca modificable a priori, por la importancia del marco y por los fantasmas que éste despierta en algunos círculos de interlocución, tal y como hemos podido observar. Por lo tanto, el 'espacio' vasco presenta una resistencia al cambio que dificulta la reflexión en torno a las necesidades de la ciudadanía en materia de servicios sociales y a la forma en que se podría responder a ellas, desplazando el centro de gravedad del discurso hacia las instituciones.

\subsection{Identidad y sujeto político}

La realidad del Sistema Vasco de Servicios Sociales se ha construido, como se ha visto, sobre una base territorial. Este elemento clave hace que la variable identitaria y su conformación en términos de ciudadanía sean también elementos importantes a la hora de determinar el relato que estamos describiendo:

Entonces, pues digamos que se consagra esa realidad, pero, a su vez, también se está consagrando la existencia de que aquí hay tres modelos y tres países. Y que entre estos tres países, esto de la asistencia social, pues bueno, tampoco es lo más importante, ¿no? (T4: 158).

Creo que es un sistema que funciona a nivel territorial. [...] Yo no vivo el Sistema Vasco de Servicios Sociales. No siento el Sistema Vasco de Servicios Sociales. Creo que debería existir, pero yo no lo vivo. Igual si hubiera avanzado un poco más la Cartera, el Mapa, o sea, si hubiéramos vivido el desarrollo de la Ley, que era lo que hacía desarrollar el Sistema Vasco de Servicios Sociales..., pues igual lo habría vivido más. Pero después de los últimos cuatro años, [en] que no ha habido nada, yo no vivo el Sistema Vasco de Servicios Sociales. No, lo siento (T3: 74).

En el análisis efectuado, hemos observado cómo tanto la propia definición de los servicios como la forma en que éstos se articulan tienen su origen y destino en el Territorio Histórico. Cada uno de los territorios tiene una manera de organizar los 
servicios, una manera de gestionarlos, incluso un presupuesto diferente que destina a su implementación. Es a partir de esta realidad, y de su contraste, desde la que parte la lectura de que existen al menos tres países en lo que se refiere a la atribución de los servicios sociales, en lugar de un único país. Y es aquí donde la lectura conecta con lo que teóricamente debería ser la definición del sujeto político.

Es cierto que esta investigación nace, de entrada, condicionada por la elección del objeto de estudio que, en este caso, es la Comunidad Autónoma de Euskadi. Es, en cierta forma, una primera acotación del sujeto político, motivada por razones de legislación común en los últimos 36 años. Se obvian, pues, deliberadamente tanto el caso de Navarra como el de los territorios vascos de Iparralde, puesto que su realidad político-administrativa dista mucho de la de la CAE, y los elementos de análisis serían, pues, muy diferentes.

Dejando al margen deliberadamente el primer debate, muy presente en la sociedad vasca, respecto al sujeto político que debería constituir o constituye la nación vasca, vemos cómo tampoco hay acuerdo en cuanto a cuál es el sujeto político que da cuerpo a lo que hoy día conocemos como CAE. 0 tal vez sí.

Los elementos que desarrollan la definición del sujeto político pueden estar relacionados con la voluntad del cuerpo social que lo compone, tal y como decía Renán en su afamada cita "la nación es un plebiscito que se formula a diario" (Renan, 2004). Pero dicha definición también puede estar determinada por el modo en que se formulan las políticas públicas y, de esta manera, la forma en que éstas determinan la condición y la definición de la ciudadanía en la práctica.

En el caso de la CAE, ¿cuáles son las políticas que pueden determinar esta condición? Pues siguiendo la definición de Marshall (1997), aquellas que afecten al desarrollo de los derechos civiles, políticos y sociales. En el caso de estos últimos, en todos aquellos ámbitos en los que la CAE es competente, a excepción de las políticas de servicios sociales, se ofrecen de forma unitaria para todo el territorio. Esta realidad nos lleva a formular dos hipótesis alternativas: o el marco que define la condición de ciudadanía y, por lo tanto, el sujeto político, es, en Euskadi, los Territorios Históricos; o en su defecto, las políticas de servicios sociales no son consideradas un elemento que se ha de tener en cuenta en esta definición.

En el primero de los casos, una conclusión semejante nos llevaría a cuestionar el concepto propio de ciudadanía vasca y a formular que una persona podría ser guipuzcoana, alavesa o vizcaína, pero no vasca, al menos en términos de determinados derechos sociales. En la segunda hipótesis, la conclusión sería que los servicios sociales no son considerados un elemento que se ha de tener en cuenta para esta definición, frente a otros, como la educación, la sanidad, la vivienda o incluso la garantía de ingresos, que sí son constitutivos de aquélla. Los servicios sociales serían, pues, políticas públicas de segunda división, cuyo desarrollo no influye a la hora de definir el marco general del resto. Serían como ese amigovio del que poder disponer en caso de necesidad, pero cuya opinión no cuenta a la hora de la verdad.

$\mathrm{Y}$, aunque reales, ninguno de estos dos escenarios parece realmente positivo. El primero, porque diluye la identidad en el territorio, creando visiones en ocasiones provincialistas, con poca visión de conjunto, a las que nuestro país no es ajeno, más allá del sistema de servicios sociales. El segundo, porque supone no tener interiorizado el discurso del cuarto pilar, la necesidad de un sistema público de servicios sociales, y porque nos retrotrae a la realidad, tantas veces descrita, de que la red de protección familiar y vecinal es perfectamente capaz de hacer frente a las necesidades derivadas de los fallos producidos en las interacciones personales.

Así pues, la indefinición del sujeto político, mal casi endémico de nuestra sociedad, se muestra de una manera todavía más virulenta en el caso del Sistema Vasco de Servicios Sociales, añadiendo un elemento más al debate en torno a su concreción.

\subsection{Financiación}

Un relato sobre la gobernanza como el que estamos construyendo siempre resultará incompleto si no cuenta con la variable financiera. El debate sobre los recursos, la posibilidad de su despliegue 0 la incidencia de la crisis económica que venimos padeciendo desde la aprobación misma de la Ley $12 / 2008$ son elementos que condicionan la mirada desde esta variable:

Entonces, creo que queda algo más difícil, yo diría, por hacer. Me atrevería a decir que los puntos de coincidencia no serían el problema. El mayor problema es quién paga y cómo se hace, más que si nos podemos poner de acuerdo en qué tipo de sistema es el deseado (P16: 53).

Una ley sin financiación es como matrimonio sin amor: para demostrar que hay amor, tiene que haber dinero; si no, está hueco (S3: 50).

El concierto económico como herramienta, y su desarrollo durante los últimos años, deja en evidencia la capacidad de la CAE para recaudar y administrar sus propios recursos ${ }^{7}$, así como la capacidad de su sistema institucional para decidir el reparto de dichos recursos en función de las necesidades del momento. En cualquier caso, queda

\footnotetext{
7 Para más información sobre el Concierto Económico, es muy recomendable la ingente obra de Pedro Luis Uriarte (2016), que se puede encontrar en 〈http://www.elconciertoeconomico.com〉.
} 
patente que el Sistema Vasco de Servicios Sociales carece hoy de los recursos que debería tener, en el escenario previsto por la propia Ley $12 / 2008$, a los efectos de conseguir la plena universalización de los servicios sociales, un escenario que, con la aprobación de la Cartera y el Plan Estratégico del Sistema, se fija a partir del 25 de diciembre de 2016.

Sucede, en cualquier caso, una extraña cuasi unanimidad a la hora de señalar una cuestión clave en el abordaje de la financiación del sistema: la ausencia de memoria económica en su aprobación. Casi todas las personas entrevistadas coinciden en que este elemento, por sí mismo, puede explicar la situación en la que el sistema se encuentra en estos momentos. Los que no lo creen así se apoyan en la falta de información y colaboración interinstitucional, o en la ausencia de esta herramienta en diciembre de 2008. En cualquier caso, es un elemento central que planea en todo el discurso en torno a la financiación del sistema, y que ha sido objeto de dimes y diretes interinstitucionales a lo largo de estos siete años de desarrollo (o no desarrollo) de la Ley 12/2008.

Hay, sin embargo, un elemento clave respecto a la financiación que se produce por el contexto en el que se ha desarrollado la última Ley de Servicios Sociales: la crisis económica. La lógica fácil podría llevar a concluir que un escenario de crisis como el que estamos viviendo es lo que impide el desarrollo del Sistema Vasco de Servicios Sociales. La mayoría de personas consultadas opinan, sin embargo, que, a pesar de la situación, con la bajada generalizada de recursos públicos y los duros años transcurridos, la crisis no es la variable principal del no desarrollo del Sistema Vasco de Servicios Sociales. Muchas voces afirman que, con la crisis, el desarrollo podría haber sido más lento, pero que se habría dado de la misma forma. Así las cosas, el elemento central de la cuestión vuelve a ser el sistema político y competencial, centrado en un concepto: el federalismo. Y dos son los elementos que se mencionan como claves para resolver esta cuestión: el Consejo Vasco de Finanzas Públicas y la Ley de Aportaciones.

En lo que se refiere al Consejo Vasco de Finanzas, su papel de órgano coordinador de las decisiones de ingresos y gastos de la CAE, al agrupar a las tres Haciendas, al Gobierno Vasco y a los municipios, a partir de la aprobación de la Ley Municipal, deja en sus manos la capacidad de distribuir más recursos en función de las necesidades detectadas por una política concreta. En el caso del Sistema Vasco de Servicios Sociales, es un movimiento imprescindible a partir del nuevo escenario que dibuja el Decreto de Cartera. La creación, para 2016, de un fondo de 10 millones de euros por parte del Consejo, con vocación de continuidad, es un paso en esta dirección, aunque todavía insuficiente.

En segundo lugar, la Ley de Aportaciones es la segunda herramienta que podría ordenar los flujos económicos entre las instituciones públicas en la
CAE. Sin embargo, tal y como también hemos visto en el análisis, está con su vigencia ya prorrogada y sin visos, en este momento, de ser renovada. La revisión de los coeficientes de reparto, a la vista de que el marco competencial es estable y las necesidades son más grandes, podría ser una de las cuestiones que su reforma debería encarar.

Por lo tanto, ninguno de los dos 'elementos correctores' está, en este momento ejerciendo la función que le ha sido encomendada. Por acción, o más bien, por omisión, están haciendo que la financiación se convierta también en una variable de importancia para la estructuración del sistema. $Y$ esto es así porque es la propia posición conservadora derivada de la organización institucional (volviendo a lo ya mencionado en los hilos anteriores) la que hace que la situación se alargue y cronifique. Así pues, la financiación se convierte en factor de importancia derivado, en forma y fondo, de la variable federal, de su forma de organización y de sus consecuencias.

\subsection{Coordinación}

En sistemas de gobernanza compleja en donde convergen distintos niveles institucionales, las relaciones intergubernamentales y, por lo tanto, la coordinación entre los distintos niveles y sus políticas, es un elemento clave a la hora de entender el relato que estamos construyendo:

El tema de la coordinación y el liderazgo son fundamentales. Todo el mundo tiene que tener claro quién es el que manda, quién manda, y nos lo tenemos que creer. Y luego, lo que tenemos es que coordinarnos; incluso aunque cambiáramos el entramado institucional, la coordinación es fundamental (T3: 163).

[Hay que] mejorar la coordinación interinstitucional, un cambio que yo creo que es más cultural que otra cosa: no sorprendernos cuando llegamos a acuerdos ( $\left.\mathrm{T}_{1}: 37\right)$.

Para examinar la coordinación, es necesario analizar el papel del Órgano Interinstitucional como el espacio que tiene encomendada esta función dentro del Sistema Vasco de Servicios Sociales. En primer lugar, conviene subrayar la falta de acuerdo respecto a la función del Órgano Interinstitucional. Entre las personas entrevistadas, están aquellas que piensan que el órgano debería ser sólo un espacio de encuentro, contraste y coordinación, pero respetando las funciones que tiene asignadas cada institución. Y están también las que ven este hecho como insuficiente y piensan que el órgano debería poder tener (si es que no las tiene ya) funciones decisorias, además de la coordinación de las políticas.

Esta falta de acuerdo en la propia definición del órgano hace también que la manera de evaluar su labor como principal herramienta para la coordinación interinstitucional se vea condicionada 
por la falta de concreción de sus funciones. De manera general, la labor del órgano como espacio de trabajo y de coordinación es juzgada positivamente. Se subrayan además las buenas relaciones entre sus participantes, y el ambiente de cordialidad y camaradería reinantes en la mayoría de las reuniones.

No sucede igual, sin embargo, a la hora de hablar del proceso de toma de decisiones dentro del órgano, siendo una misma palabra la que aparece en boca de todas las personas entrevistadas: el veto. El veto, o la necesidad de consensuar con la institución competente el desarrollo de los servicios que ésta va a ofrecer, es un planteamiento que sólo se recoge para la elaboración del Decreto de Cartera. Nació, en palabras de alguna de las personas entrevistadas, como una salvaguarda para que a nadie le fueran encomendadas competencias o desarrollos de éstas que no pudiera asumir. En la práctica, siete años después, se ha convertido en el elemento de bloqueo que ha demorado la aprobación del Decreto de Cartera y, por lo tanto, el desarrollo del sistema.

Las razones del empleo del veto pasan, en primera instancia, por la insuficiencia financiera de las instituciones que, con el desarrollo de la Ley $12 / 2008$, asumen mayores labores competenciales, principalmente los ayuntamientos. Pero también las diputaciones, a la hora de asumir ciertos servicios que no tenían o que compartían hasta este momento, aducen este tipo de dificultades. En un siguiente plano, como hemos visto, las dificultades de financiación están íntimamente ligadas con el reparto competencial y el modelo de país y, por lo tanto, en última instancia, con la variable federal. De esta forma, el veto se convierte, según todas las personas entrevistadas, en la herramienta perfecta, en la excusa más eficaz para detener y no abordar el verdadero problema de fondo, que no es ya la definición de los servicios, ni su intensidad, sino la organización del propio Sistema Vasco de Servicios Sociales.

Así pues, ¿está funcionando el Órgano Interinstitucional de forma correcta? Según las personas participantes entrevistadas, lo está haciendo. El órgano se convoca cuando corresponde, y las personas citadas acuden a la llamada. Incluso en talante y formas, las relaciones parecen ser más que correctas. ¿Pero está cumpliendo el órgano sus funciones? En este caso, la respuesta es negativa. El proceso de elaboración de normativa se eterniza y la situación de bloqueo se mantiene, incluso con cambios políticos en el Gobierno Vasco y las diputaciones forales.

\subsection{Liderazgo}

La quinta variable descrita para entender la gobernanza del Sistema de Servicios Sociales es el liderazgo. Se trata de un elemento clave en el relato de todo sistema de políticas públicas, por cuanto sirve para transmitir la dirección y las coordenadas por el que debe transitar. La idea de liderazgo, su existencia y la manera en que debería articularse también han sido cuestiones trabajadas en este ámbito:

Pues nadie y todos: nadie en particular, pero todos. Es un coliderazgo un poco caótico, y eso, por ejemplo, es un tema que tiene algo bueno, que es que todos somos parte, pero avanzamos de manera errática - si es que avanzamos, igual retrocedemos- (T2: 114).

Yo creo que funcionamos como reinos de taifas. El liderazgo es de cada institución, y quien menos tiene es el Gobierno Vasco, porque apenas gestiona, y porque, al final, tiene una función reglamentadora y de consejos de participación a nivel de País Vasco, que, al final, tiene poca eficacia en lo práctico ( $\mathrm{P}_{12}$ : 186).

¿Quién va a ejercer el liderazgo? ¿Sobre quién? Si nos han repartido las competencias, ¿luego cómo quieres ejercer liderazgo? ¡Pues no repartas las competencias! Si para ser líder te [las] tienes que quedar, es que... (T7: 84).

El concepto de liderazgo es entendido de forma distinta en función de las personas a las que se les plantee la cuestión. Existen las que, desde una lectura de la realidad institucional que compone el Sistema Vasco de Servicios Sociales en la actualidad, abogan por un liderazgo compartido entre todas las instituciones públicas competentes en la materia. Un liderazgo compartido que supondría que cada institución liderara aquella parcela de la que fuera responsable y se corresponsabilizara del rumbo general del sistema. Otras voces, partiendo también de la realidad institucional actual, abogan por un liderazgo ejercido por el Gobierno Vasco. Subrayan la necesidad de que el Gobierno asuma las tareas que tiene encomendadas en la Ley $12 / 2008$ de forma más proactiva y lidere la construcción del Sistema de Servicios Sociales, por delante del resto de los agentes, con discursos y acciones que así lo demuestren.

Visto desde cualquiera de esas dos concepciones que acabamos de describir, se puede afirmar que, en este momento, existe una carencia de liderazgo en el Sistema Vasco de Servicios Sociales. No existe liderazgo compartido, porque las instituciones competentes no se preocupan de establecer una mirada general al sistema, ni de, sea cual sea su espacio, promover una visión conjunta. Existen ejemplos en cada una de las administraciones, de creación de prestaciones propias sin visión de conjunto, o de cómo generar polémicas entre varias administraciones sin tener en cuenta la visión de conjunto o el interés general. Para que exista un liderazgo compartido, el primer paso es que haya liderazgos que quieran compartir. Ésa es la premisa que no se cumple en la actualidad. Y no existe liderazgo gubernamental porque, por un lado, sigue 
adoleciendo de las herramientas técnicas suficientes para llevarlo adelante. Pero también, y sobre todo, porque en el ordenamiento jurídico-institucional presente, tal y como está configurado, el Gobierno Vasco entiende que esa labor no le corresponde, y por lo tanto, aboga por otro tipo de liderazgos, en este caso, el liderazgo compartido mencionado anteriormente.

En definitiva, la ausencia de liderazgo está también relacionada, en última instancia, con el componente federal del sistema político y, por lo tanto, con el reparto competencial e institucional derivado de aquél. Son este reparto y esta realidad presente las que condicionan la construcción de un liderazgo sólido, incluso las que no permiten generar un liderazgo compartido. Una y otra concepción del liderazgo parten de la premisa de la voluntad de los agentes para construirlo. En el Sistema de Servicios Sociales actual, esa voluntad no existe en ninguno de los dos casos, determinada por el propio reparto competencial y territorial.

\subsection{Partidos políticos}

Los partidos políticos son otro de los elementos clave para entender la estructuración y desarrollo actual del Sistema Vasco de Servicios Sociales. El relato de los servicios sociales en Euskadi no se puede entender sin analizar la aportación de los partidos, con su posición (o no posición) en perspectiva histórica:

Creo también..., y no necesariamente en el marco de este proceso, en otros foros sí que hemos solido ver la incoherencia entre lo que defiende un partido y lo que defiende ese partido cuando está en una determinada institución. Porque, claro, luego está la realidad. Tú puedes tener a un partido diciendo una cosa, y ese partido, en el Gobierno [Vasco], o en Diputación, o en un ayuntamiento, diciendo otra cosa (C3: 81).

No, para nada, y funcionan con automatismos más de tribus urbanas; quiero decir, lo digo así de claro, porque creo que se me entiende: Bildu es como [una] tribu urbana que va a decir que sí; PP va a ser más de tribu urbana que controlen a los de fuera...; pero modelo, no [hay] (T7: 79).

Le oí a Toshack, hablando de fútbol, y decía que, cuando la Real había jugado un poco desordenada, "es que éstos corren como pollo sin cabeza". Yo creo que los partidos tienen que tener claro a dónde ir, aunque sea dentro de 50 años (S4: 63).

La conclusión es clara: los partidos políticos no tienen modelo de servicios sociales. El análisis de las propuestas formuladas en los programas electorales nos lleva a pensar que se defiende una idea general, un concepto vinculado al bienestar, pero pocas veces implementado en la realidad de los servicios sociales en Euskadi. Esta idea se confirma al contrastar lo analizado en los programas electorales con las personas entrevistadas: no existe un modelo de servicios sociales que cada partido defienda como propio y diferenciado frente a los demás.

Algunas personas afirman que, a pesar de que los partidos, como estructura, no tengan un modelo definido, hay personas que ocupan o han ocupado cargos institucionales en posiciones vinculadas con el Sistema Vasco de Servicios Sociales que tienen claro qué modelo es el que hay que implementar. Es ésta una ecuación repetida varias veces: puede que los partidos no tengan modelo, pero, sin duda, hay personas que lo tienen.

Desde esta posición, sin embargo, surge una preocupación importante: no son los partidos los que fijan la posición en el debate en torno al modelo de servicios sociales, sino la institución en la que éstos estén. El hecho de que sea la pertenencia a una u otra institución la que condicione la mirada, más allá del partido, hace que, en el mismo partido, puedan converger distintas miradas en torno a cuestiones clave del sistema. Esta situación, que no es negativa perse, puesto que puede suscitar el debate interno, genera, a la contra, una 'no posición' de los partidos con perspectiva general y una territorialización de sus planteamientos, en función del lugar y la situación en la que se encuentren.

Hay otra posición que defiende que, si no hay confrontación de modelos entre los partidos, es porque, en realidad, todos coinciden en el modelo comunitario que define la Ley $12 / 2008$ y que, por lo tanto, la discusión se produce en aquellos aspectos en los que no hay acuerdo: en el modelo de gestión, principalmente. Esta posición también admite sus dudas, al no saber qué es lo que cada uno de los partidos entiende como modelo comunitario y cómo lo traduce en sus propuestas y actuaciones. Una cosa está clara: efectivamente, hay debate en torno a los modelos de gestión, y eso es algo que todos los partidos tienen claro. Recientes debates, como el generado en torno a Kabia ${ }^{8}$ en Gipuzkoa, demuestran que, por encima del modelo de atención, en cada uno de ellos existe un criterio general respecto a cómo estos servicios deberían gestionarse.

La ausencia de modelo también determina otra cuestión fundamental: la ausencia de pugna electoral al respecto. La pugna electoral fue mencionada por varias personas durante las entrevistas como justificante de la realidad competencial y como antídoto contra las inequidades. Es decir, si la ciudadanía, con sus votos, legitima y aboga por una forma de hacer y priorizar las políticas de servicios sociales en el territorio, éste es el medio por el que

8 Instituto autónomo foral que gestiona dieciséis centros residenciales y centros de día para personas mayores o en situación de dependencia, de titularidad municipal. El debate sobre su creación se produjo en Juntas Generales el 22 y 23 de octubre de 2014, y en él se pudieron escuchar argumentos, en líneas generales, referidos al modelo de gestión de las políticas públicas, más que al sistema de servicios sociales en particular. 
también habría que corregirlo. Este argumento, sin embargo, choca con la realidad que acabamos de describir: la política de servicios sociales no forma parte de la pugna electoral. Y no lo hace por la inexistencia de elementos de debate en los partidos políticos. 0 porque no se considera un tema de gran interés para ello. En cualquier caso, no forma parte de la arena electoral.

Así pues, la falta de modelo de los partidos políticos, y todo lo que ello supone, es un factor importante que determina también la estructura del Sistema Vasco de Servicios Sociales. La ausencia de modelo determina la ausencia de pugna electoral. En consecuencia, también la dirección y el rumbo que el Sistema pueda adquirir queda determinado por las personas que gestionan cada una de las instituciones, con criterio, pero determinadas más por el peso de las propias instituciones que por el modelo que habría que implementar respecto al Sistema en su globalidad.

Los partidos son agentes políticos de primer orden en una sociedad democrática representativa, como la nuestra, y la ausencia de discursos o de ideas clave respecto al Sistema determina de manera importante el resultado de éste.

\subsection{Conocimiento}

La última variable que construye nuestro particular relato hace referencia al conocimiento, en dos claves distintas: el conocimiento que la población tiene respecto al Sistema Vasco de Servicios Sociales; y el conocimiento científico, elemento básico para la consolidación y extensión de toda disciplina.

Quiero decir, ¿qué son los servicios sociales? ¡Pero si la mayoría de la gente no [lo] sabemos, pero ni nosotros mismos! ( $\left.T_{5}: 77\right)$.

Hoy en día no tenemos un producto llamado servicios sociales que la gente quiera. Es decir, si a ti ahora se te pone un dolor muy fuerte en el pecho, y llega una vecina y te dice que a ella una vez le pasó algo parecido, luego llega un curandero que te dice que te tomes una cebolla y, a la vez, llega uno de Osakidetza con la bata blanca, tú distingues perfectamente lo que te va dar cada uno. Tú ves qué te va a dar tu amigo o persona cercana, el profesional no acreditado o el producto del que tiene la bendición del sistema. Sin embargo, te llama tu madre y te dice "mira, me han operado de la cadera y tengo que volver a casa, pero no puedo volver sola, necesito un apoyo"..., pues la gente prefiere una señora rumana a la que paga una miseria y la tiene medio de interna, en lugar de una auxiliar de ayuda a domicilio bendecida por los servicios sociales del ayuntamiento. En parte eso es así, porque tampoco es verdad que el producto sea tan diferente... Hay una cosa que es del producto, porque está ahí (C4: 56).
Es llamativa la fragilidad de ese entramado de agentes... Prácticamente nos juntamos en un txoko y estamos todos allí. Falta masa crítica de representación de intereses, de preparación de propuestas... Sin embargo, la envergadura del sistema daría para más. Hay una especie de minimización de la parte... Tampoco no lo había expresado de esta forma (C4: 59$)$.

Decía una de las personas entrevistadas que, si juntáramos a todas las personas con criterio y reflexión en torno al Sistema de Servicios Sociales en Euskadi, podrían entrar en un $t x o k o^{9}$. Y no le falta razón.

De nuestro análisis se extrae que el conocimiento de la ciudadanía respecto al Sistema Vasco de Servicios Sociales es escaso. Sin embargo, este elemento es clave para la construcción del sistema y explica, junto con los ya mencionados, su situación actual.

Éste es un elemento importante por dos razones. En primer lugar, porque el reconocimiento social es una clave fundamental para la extensión de todo sistema de políticas públicas. La ciudadanía no sólo debe conocer las prestaciones y servicios que forman parte él; debe también reconocerse en ellas. Y esto hará que la presión en torno a la calidad de los servicios, al modelo existente, crezca, introduciendo así la variable en la agenda política. Pero no es lo que sucede en la actualidad.

La falta de visibilización del sistema como conjunto puede ser una de las causas para este desconocimiento. La ciudadanía es capaz de identificar qué servicios ofrece la diputación y cuáles el ayuntamiento, pero no que todos forman parte de un mismo sistema, con un mismo terreno de juego. Volvemos a la variable federal. En segundo lugar, la complejidad del concepto 'servicios sociales' y el no acuerdo existente en torno a la necesidad a la que responde también puede ser una de las razones del desconocimiento sobre el sistema.

Esto nos lleva a la segunda acepción de la variable: el conocimiento científico. Ha sido también una de las cuestiones expuestas por las personas entrevistadas, y no es menor en importancia a las mencionadas anteriormente. La falta de un corpus teórico de la disciplina y la falta de conocimiento científico en aquellos espacios dedicados para ello, principalmente universidades y fundaciones dedicadas a la investigación, son elementos que deberían influir de manera decisiva en la estructuración del Sistema. Esta falta de corpus y de recorrido teórico está relacionada, por un lado, con lo novedoso del ámbito. En comparación con los sistemas educativo y sanitario, el de servicios sociales ha llegado tarde y con mucho debate

9 Denominación de las sociedades gastronómicas en Bizkaia. Una sociedad gastronómica es un club privado de hombres (en su origen, aunque ahora también las hay mixtas) en el que se juntan para cocinar y comer. De mucha tradición en Euskadi. 
todavía pendiente en términos de necesidades y tipos de respuesta. Por otro lado, también tiene que ver con las personas y los perfiles que construyen esta disciplina. El sistema de servicios sociales ha estado históricamente vinculado con el perfil del trabajador y trabajadora social. Un perfil éste que, tradicionalmente ha entendido la realidad de la investigación como un complemento a su actividad principal: la intervención ${ }^{10}$. Y esto se visualiza como dificultad a la hora de construir la disciplina a la que va más íntimamente ligado. También es cierto que el estatus que el trabajo social ha tenido hasta el momento no le ha permitido incorporarse a las tareas vinculadas a la gestión e investigación de políticas.

En cualquier caso, por una razón o por otra, es constatable la metáfora del txoko, y las universidades vascas no son ajenas a esta realidad. También desde la Universidad será necesaria una reflexión en torno a los porqués de la situación y al papel que le corresponde en la construcción del conocimiento científico en materia de servicios sociales y, por lo tanto, de su estructuración como sistema.

Así pues, el conocimiento es también una variable fundamental para entender la configuración y estructuración del Sistema Vasco de Servicios Sociales.

\section{Consideraciones finales}

El relato de la gobernanza del Sistema Vasco de Servicios Sociales se construye tirando de los hilos de la madeja que ha ido formado el conjunto de agentes, instituciones y personas que forman parte de él. Una madeja compleja, enrollada y poco clara que comienza a vislumbrar unos claros de luz, pero que sigue teniendo muchos nudos por soltar. Es la 'arena' (Lowi, 1964) de la política de servicios sociales vasca, en la que esos actores interactúan.

En este artículo, hemos dibujado un relato que configura una gobernanza compleja. Un relato cuyos elementos hay que tomar en consideración para construir la realidad de un sistema de servicios sociales joven, indeciso y fragmentado. Un sistema, el cuarto pilar del Estado de bienestar, que no ha tenido el desarrollo de los otros tres pilares, ni tampoco su reconocimiento social. ¿Pero cómo se construye el sistema en su día a día? ¿Cuáles son sus elementos explicativos principales?

El elemento territorial constituye, tal y como hemos visto, el primero de los atributos que conforman nuestro relato. No es posible entender ni interpretar la realidad del sistema si no se entiende previamente el peso que el factor foral tiene en su construcción. Un factor ausente en la implementación en la CAE

${ }_{10}$ Esta realidad ha cambiado en los últimos años, a partir del reconocimiento del grado en Trabajo Social como equiparable a los del resto de disciplinas, y de la producción de investigación y tesis doctorales en este ámbito. del resto de sistemas de bienestar. Un factor que responde, por una parte, a la configuración legal del año 1983, motivada por la poca importancia otorgada a este ámbito, y por otra, al recorrido de la senda institucional (path dependency) que ha ido afianzando el modelo. Sea por lo que fuere, el peso de la división institucional y el reparto de competencias es grande en el relato del sistema, condiciona su día a día y no es probable, además, que se vaya a modificar a corto ni medio plazo.

Este primer elemento nos transporta irremediablemente al segundo: existen tres países dentro de la CAE a la hora de implementar el Sistema. Porque cada uno de los Territorios Históricos parte de una realidad concreta. Y porque desarrolla después un modelo concreto. Como resultado de este proceso, la propia definición de ciudadanía es cuestionada y surge la pregunta (abierta a la discusión) de cómo ha de definirse y sobre qué sujeto político se realiza.

El tercero de los elementos es, inevitablemente, el financiero. En un escenario territorializado y con múltiples instituciones en juego; no sólo es importante la suficiencia financiera del sistema en su conjunto, sino la capacidad de regular los flujos financieros interinstitucionales $y$, finalmente, la respuesta de cada una de las instituciones, con sus propios recursos, a aquello que tiene encomendado. Este elemento ha cobrado protagonismo por el debate surgido de la bajada de ingresos derivada de la crisis económica que estamos padeciendo durante los últimos años. Pero es un elemento que influye, con crisis o sin ella, por acción u omisión del Consejo Vasco de Finanzas y los flujos que en él se deciden. El factor territorial gravita alrededor de este elemento, al que la dispersión institucional tampoco ayuda en demasía.

Partiendo de este escenario, dos elementos ayudan (o no) a empastarlo: la coordinación y el liderazgo. La coordinación como elemento necesario de tracción interinstitucional. Como aquello imprescindible para que un sistema de características intergubernamentales y que adolece de falta de voluntad, como éste, funcione. Y un liderazgo global tan imprescindible como ausente. Porque cada cual lidera con más o menos éxito la institución de la que forma parte. Pero siendo esto así, ¿quién lidera el sistema? ¿Quién lo siente como propio?

Los partidos políticos, elementos claves en la legitimación de los sistemas de políticas públicas, vehículos por donde puede transitar el debate público, en este asunto quedan sumergidos por la visión de las instituciones que impregna la posición de las personas que las representan. Hace falta debate y posición. Es absolutamente necesario trabajar en la esfera del conocimiento, para fijar modelos y elementos que puedan incidir en el debate público. Pero no sólo los partidos. Las universidades deben también trabajar con más ahínco en la investigación y transferencia de los servicios sociales. Y las personas trabajadoras, en 
la sistematización. Para seguir dando forma a un ámbito de difícil definición, pero necesario en su articulación.

Así pues, el relato de la gobernanza del Sistema Vasco de Servicios Sociales se completa entendiendo que, a este sistema fragmentado, debemos añadir:

- La territorialidad (foralidad) como elemento de diversidad y diferencia.

- El concepto de ciudadanía construido sobre este mismo criterio (¿ciudadanía desde dónde?).

- Una financiación institucionalmente fraccionada, que genera dificultades de gestión, acentuadas en épocas de baja recaudación.

- Instrumentos para su coordinación teórica, pero dificultades evidentes para llevarla a la práctica.

- Ausencia de liderazgo claro y compartido.
- Un modelo que se construye desde la posición institucional, más que desde perspectivas ideológicas.

- Un modelo que necesita definir teóricamente su marco de actuación.

A la luz de estos elementos, si queremos ponernos a desatar los nudos de la madeja, no habrá más remedio que poner en marcha un debate público serio y ordenado. Un debate en el que participen los agentes del sistema en torno a su configuración y extensión. También los partidos políticos, con una posición trabajada previamente, deberían tomar parte. Y sobre todo, si queremos que la legitimación y valoración de la ciudadanía aumente, habrá que hacerla participe de todos los elementos del sistema, desde la definición del objeto hasta la identificación del propio sistema. Todos éstos son elementos clave para avanzar en la definición de un sistema maduro, estable y con proyección de futuro. 


\section{Referencias bibliográficas}

ADELANTADO, J.; y JIMÉNEZ, A. (2003): “Las políticas de servicios sociales en las comunidades autónomas", en GALLEGO, R.; GOMÁ, R.; y SUBIRATS, J. (eds.), Estado de bienestary comunidades autónomas, Madrid, Tecnos.

ALEMÁN, C.; ALONSO SECO, J. M.; y GARCíA SERRANO, M. (2011): Servicios sociales públicos, Madrid, Tecnos.

ARRIETA FRUTOS, F. (2015): “Factores determinantes de la complejidad del Sistema Vasco de Servicios Sociales y sus efectos en la provisión territorial de servicios" [tesis doctoral], Universidad de Deusto, Facultad de Ciencias Sociales y Humanas.

AZNAR, M. (1994): “La legislación autonómica de acción social. Análisis jurídico", en CASADO, D. (ed.), $V$ informe sociológico sobre la situación social en España, Madrid, Fundación Foessa.

CASADO, D. (2007): “Apunte histórico de la construcción de los servicios sociales", en CASADO, D.; y FANTOVA F. (eds.), Perfeccionamiento de los servicios sociales en España: informe con ocasión de la Ley sobre Autonomía y Dependencia, Madrid, Cáritas Española.

DUQUE, T. (2012): "Redescubrimiento de los servicios sociales de atención primaria: hacia un (nuevo) modelo de atención personal y comunitaria", Zerbitzuan, n-0 52, págs. $23-44$ [/http://dx.doi. org/10.5569/1134-7147.52.02>].

ESPAÑA (2013): "Ley 27/2013, de 27 de diciembre, de racionalización y sostenibilidad de la Administración Local”, Boletín Oficial del Estado, n- 312, 30-12-13, págs. 106.430106.473 [<https://www.boe.es/diario_boe/txt. php?id=BOE-A-2013-13756)].

- (2012): “Real Decreto-Ley 20/2012, de 13 de julio, de Medidas para Garantizar la Estabilidad
Presupuestaria y de Fomento de la Competitividad", Boletín Oficial del Estado, no $168,14-7-12$, págs. 50.428-50.518 [khttp://www.boe.es/diario_boe/txt. php?id=BOE-A-2012-9364'].

- (2006): “Ley 39/2006, de 14 de diciembre, de Promoción de la Autonomía Personal y Atención a las Personas en Situación de Dependencia", Boletín Oficial del Estado, ํㅜ 299, 15-12-06, págs. 44.142-44.156 [<http://www.boe.es/ diario_boe/txt.php?id=BOE-A-2006-21990〉].

- (1985): "Ley 7/1985, de 2 de abril, Reguladora de las Bases del Régimen Local”, Boletín Oficial del Estado, $\mathrm{n} 0$ 80, 3-4-85, págs. 8.9458.964 ['http://www.boe.es/diario_boe/txt. php?id=BOE-A-1985-5392>].

- (1979): "Ley Orgánica 3/1979, de 18 de diciembre, de Estatuto de Autonomía para el País Vasco", Boletín Oficial del Estado, no 306, 22-12-79, págs. 29.357-29.363 [<http://www.boe.es/ diario_boe/txt.php?id=BOE-A-1979-30177)].

- (1978): “Constitución Española”, Boletín Oficial del Estado, 29-12-1978, no 311, págs. 29.31329.424 [<https://www.boe.es/buscar/act. php?id=BOE-A-1978-31229>].

ETXEZARRETA, E. (2012): Gizarte ekonomiaren aukera zaintzen krisiaren aurrean: Gipuzkoako pertsona nagusien alorra aztergai, Universidad del País Vasco [rhttp://www.euskara.euskadi. net/appcont/tesisDoctoral/PDFak/Enekoitz Etxezarreta_TESIA.pdf $\rangle]$.

FANTOVA, F. (2009): “Marco conceptual, normativo y estratégico para una política foral de servicios sociales en Gipuzkoa: hacia una comprensión, encaje y desarrollo de los servicios públicos en el ámbito de los servicios sociales”, 
en DEPARTAMENTO DE POLITICA SOCIAL (ed.), Ponencias el proceso de reflexión / Hausnarketa prozesuaren txostenak, DonostiaSan Sebastián, Diputación Foral de GipuzkoaGipuzkoako Foru Aldundia.

- (2006): "Algunos elementos para un análisis de las políticas sobre servicios sociales en el País Vasco", Zerbitzuan, no 40, págs. 7-20 [<http:// www.zerbitzuan.net/documentos/zerbitzuan/ Elementos\%2opara\%20un\%2oanalisis\%20 de\%2olas\%2opoliticas\%2osobre\%20 servicios\%2osociales.pdf〉].

GALLASTEGUI, M. C.; y GALLASTEGUI, I. (1986): Un análisis económico de la Ley de Territorios Históricos, Donostia, Eusko Ikaskuntza.

GALLEGO, R.; GOMÁ, R.; y SUBIRATS, J. (2003): Estado de bienestar y comunidades autónomas, Madrid, Tecnos.

GALLEGO, R.; y SUBIRATS, J. (2011): Autonomies i desigualtats a Espanya: percepcions, evolució social y polítiques de benestar, Barcelona, Generalitat de Catalunya.

GOIKOETXEA, J. (2012): "Nationalism and democracy in the Basque Country (1979-2012)", Ethnopolitics [iFirst] [<http://dx.doi.org/10.1080/17449057.2 012.711049>].

LOWI, T. J. (1964): “American business, public policy, case studies, and political theory", World Politics, vol. $4, \mathrm{n}^{0} \mathrm{16}$, págs. $677-715$.

MARSHALL, T. H. (1997): “Ciudadanía y clase social”, Revista de Investigaciones Sociológicas, $\mathrm{n}^{\circ} \mathbf{7 9}$, págs. 297-344.

NOVO, A. (2010): La excepcionalidad del modelo federal foral vasco, Valencia, Tirant lo Blanch.

PAÍS VASCO (2016): “Ley 2/2016, de 7 de abril, de Instituciones Locales de Euskadi”, Boletín Oficial del País Vasco, no 70, 14-4-16 [rhttps:// www.euskadi.eus/y22-bopv/es/bopv2/ datos/2016/04/1601544a.shtml>].

- (2015): “Decreto 185/2015, de 6 de octubre, de Cartera de Prestaciones y Servicios del Sistema Vasco de Servicios Sociales", Boletín Oficial del País Vasco, nํㅜ 206, 29-10-15, págs. 1-87 [<https:// www.euskadi.eus/y22-bopv/es/bopv2/ datos/2015/10/1504561a.shtml>].

- (2014): "Circular de 11 de marzo de 2014, de la Directora de Relaciones con las Administraciones Locales y Registros Administrativos, referente al sistema de ordenación de las competencias municipales y al régimen foral vasco, tras la entrada en vigor de la Ley $27 / 2013$, de 27 de diciembre, de Racionalización y Sostenibilidad de la Administración Local”, Boletín Oficial del País Vasco, nํ56, 21-3-14, págs. [khttps:// www.euskadi.eus/y22-bopv/es/bopv2/ datos/2014/03/1401377a.shtmls].

- (2011): "Decreto 225/2011, de 26 de octubre, del Observatorio Vasco de Servicios Sociales", Boletín Oficial del País Vasco, n- 215, 14-11-11, págs. [rhttps://www.euskadi.eus/y22-bopv/ es/bopv2/datos/2011/11/1105502a.shtml'].

- (2010): “Decreto 101/2010, de 30 de marzo, del Órgano Interinstitucional de Servicios Sociales", Boletín Oficial del País Vasco, no 71, 19-4-10 [<https://www.euskadi.eus/y22-bopv/es/ bopv2/datos/2010/04/1002076a.shtml/].

- (2010): “Decreto 238/2010, de 14 de septiembre, de la Alta Inspección en materia de Servicios Sociales", Boletín Oficial del País Vasco, no 189, 30-3-10 [rhttps://www.euskadi.eus/ y22-bopv/es/bopv2/datos/2010/09/1004510a. shtmls].

- (2008): “Ley 12/2008, de 5 de diciembre, de Servicios Sociales", Boletín Oficial del País Vasco, n- 246, 24-12-08, págs. 31.840-31.924 [rhttps://www.euskadi.eus/r47-bopvapps/es/ bopv2/datos/2008/12/0807143a.shtml〉].

- (2001): “Decreto 155/2001, de 30 de julio, de Determinación de Funciones en Materia de Servicios Sociales", Boletín Oficial del País Vasco, no 165, 27-8-01 [rhttps:// www.euskadi.eus/y22-bopv/es/bopv2/ datos/2001/08/0104794a.shtml〉].

- (1996): “Ley de Servicios Sociales 5/1996”, Boletín Oficial del País Vasco, no 218, 12-11-96, págs. 17.57817.599 [khttps://www.euskadi.eus/y22-bopv/ es/bopv2/datos/1996/11/9605350a.shtml'].

- (1983): “Ley 27/1983, de 25 de noviembre, de Relaciones entre las Instituciones comunes de la Comunidad Autónoma y los órganos forales de sus Territorios Históricos", Boletín Oficial del País Vasco, no 182, 10-12-83, págs. 4.132-4.150 [<https://www.euskadi.eus/y22-bopv/es/ bopv2/datos/1983/12/8302316a.shtmls].

RENAN, E. (2004): “Zer da nazioa?”, en ANTXUSTEGI, E. (ed.), Filosofia politikorako irakurketak, Bilbao, Universidad del País Vasco.

RODRÍGUEZ CABRERO, G. (2004): El Estado de Bienestar en España: debates, desarrollo y retos, Madrid, Fundamentos.

SACANELL, E. (2009): La situación actual de los servicios sociales en Euskadi [conferencia], Donostia-San Sebastián, Cursos de Verano de la Universidad del País Vasco.

SOBREMONTE, E. (2009): Deshaciendo la madeja de los servicios sociales [conferencia], Donostia-San Sebastián, Cursos de Verano de la Universidad del País Vasco.

SOTELO, H. (2005): "Marco normativo de los servicios sociales en la CAPV y propuesta de reordenación”, en VV.AA. (ed.), Situación y perspectivas del sistema público de servicios sociales en la Comunidad Autónoma del País Vasco, Vitoria-Gasteiz, Servicio Central de Publicaciones del Gobierno Vasco.

TRIBUNAL CONSTITUCIONAL (2016): “Pleno. Sentencia 41/2016, de 3 de marzo de 2016. Recurso de inconstitucionalidad 1792-2014", Boletín Oficial del Estado, no 85, 8-4-16, págs. 24.98825048 ['https://www.boe.es/diario_boe/txt. php?id=BOE-A-2016-3407>].

URIARTE, P. L. (2016a): El Concierto Económico Vasco: una visión personal [<http://www. elconciertoeconomico.com/descargate-laobra/s].

- (2016b) Nuestro Concierto: claves para entenderlo [rhttp://www.elconciertoeconomico.com/ descargate-la-obra/s]. 